\title{
Determinação das glicemias capilar e venosa com glicosimetro versus dosagem laboratorial da glicose plasmática
}

\author{
Determination of capillary blood glucose and venous blood glucose with a glucometer versus laboratory \\ determination of venous plasma glucose
}

Caio Mauricio Mendes de Cordova'; Jéssyca Pereira Valle²; Celina Noriko Yamanaka ${ }^{3}$; Maurício Mendes de Cordova ${ }^{4}$

\begin{abstract}
unitermos
resumo

Glicemia

Introdução e objetivos: Diabetes mellitus (DM) é a mais importante patologia que envolve o pâncreas

Diabetes mellitus

endócrino, sendo uma das principais causas de morbidade e mortalidade na população geral. O objetivo deste trabalho foi avaliar a determinação da glicemia em diferentes tipos de amostras e metodologias.

Automonitoramento da

glicemia

Métodos: Utilizando um equipamento Accu-Check Advantage (Roche), foi avaliada a glicemia em amostras de sangue capilar (GCG) e de sangue venoso (GVG), e a dosagem da glicemia venosa em

Glicosímetros plasma (GVE) foi realizada por método enzimático de rotina. Resultados e conclusão: Foi observada boa

Glicemia capilar correlação entre a GCG e a GVG $(r=0,8742)$. Entretanto, houve diferença significativa entre a GCG e a $\operatorname{GVE}(r=0,6543)$ e entre a GVG e a GVE $(r=0,5038)(p<0,001)$. Essa diferença é maior considerando-se apenas os pacientes normoglicêmicos. $O$ fato de haver diferentes estudos com resultados conflitantes entre si não depende somente da marca ou série específica do glicosímetro, já que uma mesma marca pode apresentar resultados inconsistentes em diferentes estudos.
\end{abstract}

abstract

Introduction and objectives: Diabetes mellitus is the most important pathology that affects the endocrine pancreas and one of the main causes of morbidity and mortality among the general population. The aim of this work was to evaluate the correlation of blood glucose determination in different types of samples and methodologies. Methods: Using an Accu-Check Advantage glucometer (Roche), it was determined the blood glucose levels in capillary (CBG) and venous (VBG) samples, and plasma venous glucose was determined by routine enzymatic methodology (EVG). Results and conclusion: It was observed a good correlation between $C B G$ and VBG $(r=0.8742)$. However, there was a significant difference between $C B G$ and EVG $(r=0.6543)$ and between VBG and EVG $(r=0.5038)(p<0.001)$. These differences are even more substantial considering only normoglycemic patients. The existence of different studies with inconsistent results does not depend only on the glucometer brand or its specific series, as the same device may present inconsistent results in different studies. key words

Blood glucose

Diabetes mellitus

Monitoring system

Glucometers

Capillary glycemia

4. Bacharel em Bioquímica pela Universidade Federal do Paraná (UFPR). 


\section{Introdução}

O diabetes mellitus (DM) é a mais importante patologia que envolve o pâncreas endócrino, sendo uma das principais causas de morbidade e mortalidade na população geral. Estima-se que no Brasil existam 5 milhões de indivíduos portadores de DM, dos quais metade desconhece o diagnóstico. Desse total, $90 \%$ são do tipo 2 (DM2), ou não-dependentes de insulina, $8 \%$ a $9 \%$ do tipo 1 (DM1), ou dependentes de insulina, de origem autoimune, e $1 \%$ a $2 \%$ portadores de diabetes secundário ou associado a outras síndromes ${ }^{(11)}$.

No Brasil é elevada a incidência de complicações crônicas devido principalmente à existência de poucos programas de educação e de capacitação profissional em diabetes e de recursos escassos para prevenção e controle da moléstia. Ações conjuntas do Ministério da Saúde (MS) e da Sociedade Brasileira de Diabetes (DBD) têm melhorado a situação nos últimos anos, mas ainda assim a morbimortalidade por diabetes é elevada(11).

A prevenção e o controle de DM são clinicamente factíveis por meio da identificação e do tratamento de indivíduos sob alto risco de desenvolver diabetes, de controle clínico daqueles já acometidos e de detecção e tratamento precoce das complicações. Um dos grupos sob risco de desenvolvimento de diabetes que tem recebido crescente atenção é o da tolerância diminuída à glicose, presente em $7,8 \%$ dos adultos de capitais brasileiras ${ }^{(5)}$.

O monitoramento laboratorial dos níveis de glicose é extremamente importante para acompanhar o tratamento e prevenir as complicações de DM. Com a difusão dos apareIhos para aferição da glicemia capilar pelo próprio paciente, frequentemente em seu próprio domicílio, têm surgido muitos questionamentos sobre as diferenças de resultados encontrados pelo paciente e aqueles determinados pelo laboratório. A literatura internacional oferece dados conflitantes sobre avaliações de diferentes métodos.

Sendo o DM uma doença crônica, que requer alterações no estilo de vida, considerada problema de saúde pública prevalente em estado crescente, responsável por várias complicações, considera-se a importância desse estudo no sentido de ampliar os conhecimentos dos profissionais da saúde para a educação do paciente diabético com relação aos diferentes métodos de avaliação de dosagem da glicemia. Com o advento da monitoração dos níveis glicêmicos pelos próprios pacientes e o uso de glicosímetros, temos observado crescente questionamento por parte dos mesmos quanto aos resultados obtidos no laboratório, por requisição médica, da dosagem da glicemia plasmática por métodos enzimáticos de rotina. Alguns pacientes chegam ao extremo de, no momento da coleta do sangue venoso no laboratório, solicitar que uma gota seja colocada no seu glicosímetro particular para comparar os resultados posteriormente. Dada a falta de consenso sobre a validade da comparação entre esses três tipos de resultado de glicemia, o objetivo deste trabalho foi determinar sua correlação e discutir seu impacto no monitoramento do paciente diabético.

\section{Material e métodos}

\section{Pacientes}

Neste estudo foram avaliados pacientes que procuraram o laboratório de análises clínicas do ambulatório universitário da Universidade Regional de Blumenau (FURB) de janeiro a agosto de 2009, com requisição médica para determinação da glicemia em jejum. Foram excluídos do estudo aqueles que não aceitaram fornecer uma amostra de sangue capilar adicional para a realização das análises. Analisaram-se amostras de 55 pacientes, sendo 37 do sexo feminino e 18 do masculino, com idades entre 18 e 79 anos.

\section{Amostras}

Foram obtidas amostras de sangue venoso por punção da veia basílica ou cubital média com seringa hipodérmica não reutilizável. Cerca de $3 \mathrm{ml}$ de sangue foram colocados em tubo contendo fluoreto de sódio, para evitar o consumo da glicose, e homogeneizados. Em seguida, o tubo foi centrifugado por 5 minutos a $2.500 \mathrm{rpm}$ para separação do plasma, que foi armazenado a $4^{\circ} \mathrm{C}$. As dosagens da glicose nas amostras de plasma foram realizadas no mesmo dia da coleta. As amostras de sangue capilar foram obtidas por punção da polpa do dedo médio, com o uso de lanceta não reutilizável, após antissepsia da região com etanol a $70 \%$. O sangue obtido no local da punção foi imediatamente aspirado pela fita reativa do glicosímetro.

\section{Dosagem da glicose}

A glicemia em jejum foi realizada primeiramente com a amostra de sangue capilar mediante o glicosímetro AccuCheck Advantage (Roche Diagnóstica Brasil). Da mesma forma, o sangue venoso homogeneizado obtido por venopunção também foi utilizado para a determinação da glicose 
pelo glicosímetro. Por fim, o plasma obtido do sangue venoso após centrifugação foi usado para determinar a glicose por método enzimático colorimétrico com a enzima glicose oxidase (Biosystems, Barcelona, Espanha) em um equipamento BTS 310, de acordo com as especificações dos fabricantes. O laboratório apresenta certificação "Excelente" pelo Programa Nacional de Controle de Qualidade (PNCQ).

\section{Avaliação dos métodos}

Os dados obtidos foram tabulados de acordo com os três resultados possíveis: glicemia em sangue capilar com o glicosímetro (GCG), glicemia em sangue venoso com glicosímetro(GVG) e glicemia venosa em plasma com o método colorimétrico enzimático (GVE). Os dados foram ainda classificados em dois grupos: glicemia $<5,55 \mathrm{mmol} / \mathrm{l}$ (100 mg/dl) e glicemia $\geq 5,55 \mathrm{mmol} / \mathrm{l}$, de acordo com o resultado obtido pela dosagem plasmática com o método colorimétrico. A comparação entre os métodos de dosagem da glicemia foi realizada de acordo com as recomendações do Clinical and Laboratory Standards Institute (CLSI) ${ }^{(10)}$. Coeficientes de correlação, erro constante e erro proporcional foram obtidos mediante a análise de regressão de Deming, e o gráfico de Bland-Altman foi construído com o auxílio dos programas Excel (Microsoft, EUA) e Analyze-it (UK). Os dados referentes a média, mediana, desvio padrão e significância estatística foram calculados com o auxílio do programa Excel, pelo teste $t$ unicaudal com variância homoscedástica. Para determinar a imprecisão dos métodos foram analisadas 20 dosagens diárias consecutivas da glicose no soro controle interno no Programa Nacional de Controle de Qualidade para o cálculo do coeficiente de variação, pelo método enzimático colorimétrico. Para avaliar a imprecisão da determinação da glicose pelo glicosímetro, dada a indisponibilidade de um controle de glicose estável em sangue total, foram feitas 20 dosagens consecutivas, no mesmo dia, da glicose em uma amostra de sangue total obtida aleatoriamente no laboratório. Os limites recomendados de desempenho intralaboratorial são um coeficiente de variação (imprecisão) $\leq 3,3 \%$, com um erro (bias) $\leq 2,5 \%$ e um erro total $\leq 7,9 \%{ }^{(12)}$.

\section{Resultados}

Analisando os resultados dos pacientes obtidos pelas três diferentes metodologias, foi obtida uma média da GCG de 5,64 mmol// com desvio padrão de 0,87 e me- diana de 5,49 mmol/l. A GVG resultou numa média de $5,83 \mathrm{mmol} / \mathrm{l}$, com desvio padrão de 0,76 e mediana de $5,72 \mathrm{mmol} / \mathrm{l}$. Da mesma forma, a GVE apresentou média de $5,02 \mathrm{mmol} / \mathrm{l}$ com desvio padrão de 0,73 e mediana de $4,94 \mathrm{mmol} / \mathrm{l}$ (Tabela). Os coeficientes de variação da dosagem da glicose pelo método enzimático colorimétrico e pelo glicosímetro foram, respectivamente, $3,1 \%$ e $1,9 \%$.

Avaliando a comparação dos resultados pelos diferentes métodos, observa-se que existe boa correlação entre a determinação da glicemia capilar pelo glicosímetro e a utilização de sangue venoso para o estabelecimento da glicemia no mesmo equipamento $(r=0,8742)$, com resultados muito próximos entre si $(p>0,05)$ (Figura 1A).

Entretanto, utilizando equipamentos diferentes, como na comparação entre a GVG e a GVE, observa-se correlação bastante pobre $(r=0,5038 ; p<0,001)$ (Figura 1B). Da mesma forma, comparando-se a GCG, que pode ser obtida pelo próprio paciente, com a GVE, existe pouca correlação entre os dois resultados $(r=0,6543 ; p<0,001)$

\section{(Figura 1C).}

O gráfico das diferenças de Bland-Altman demonstra a baixa correlação entre GCG e GVE, com um erro (bias) médio de $-11,5 \%$ (-36,4 a +13,4\% e intervalo de confiança [IC] de 95\%), e entre GVG e GVE, com erro médio de $-15,1 \%(-42,6$ a $+12,5 \%$ com IC de $95 \%)$. O erro médio da GVG em relação à GCG é de +3,6\% (-11,7 a +18,9\% com um intervalo de confiança de $95 \%$ ) (Figura 2).

Essas diferenças são ainda mais evidentes separandose os pacientes de acordo com o seu nível glicêmico no plasma pelo método enzimático, considerando uma glicemia normal $(<5,55 \mathrm{~mol} / \mathrm{l})$ ou alterada $(\geq 5,55 \mathrm{mmol} / \mathrm{l})$. Observou-se que as correlações entre a GVG e a GVE ( $r=$ $0,0304)$, entre a GCG e a GVG $(r=0,7473)$ e entre a GCG e a GVE $(r=0,2708)$ ficam ainda piores considerando-se apenas os indivíduos normoglicêmicos $(p<0,001)$.

Considerando apenas os pacientes com glicemia alterada ( $\geq 5,55 \mathrm{mmol} / \mathrm{l})$, apesar de a pequena amostragem poder prejudicar o poder do teste estatístico, a correlação entre a GVG e a GVE melhora ligeiramente $(r=0,3617)$, embora ainda haja diferença significativa $(p=0,04)$, assim como na correlação entre a GCG e a GVE $(r=0,416)$. Nesse grupo de pacientes, a correlação entre a GCG e a GVG é bastante aceitável $(r=0,9572 ; p=0,41)$. O gráfico dos resultados normalizados derivado da regressão de Deming ilustra claramente que os valores normoglicêmicos se desviam mais do resultado "verdadeiro" estimado do que os valores hiperglicêmicos (Figura 3). 


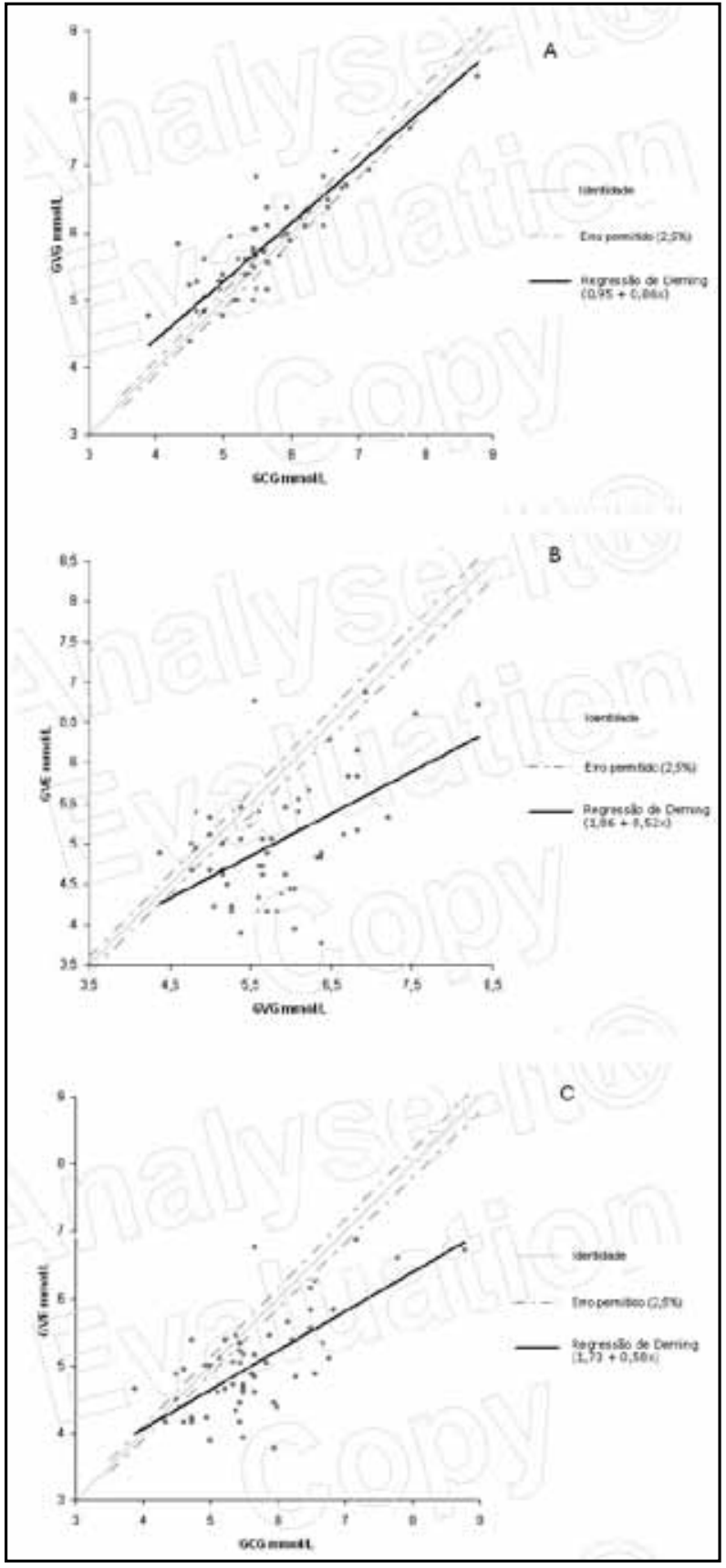

Figura 1 - A: Correlação pela análise de regressão de Deming entre os resultados da GCC e da GVG; B: entre a GVG e GVE; C: GCG e a GVE

GCC: glicemia capilar determinada pelo glicosímetro; GVG: glicemia venosa determinada pelo glicosímetro; GVE: glicemia venosa determinada em plasma pelo método enzimático.

\section{Discussão}

Com o advento da terapêutica capaz de trazer o controle glicêmico para níveis de quase normoglicemia, as metas pretendidas para esse controle tornaram-se mais estritas. Dois experimentos clínicos de grande porte, em pacientes com diabete tipos 1 e 2, evidenciaram a importância do al-

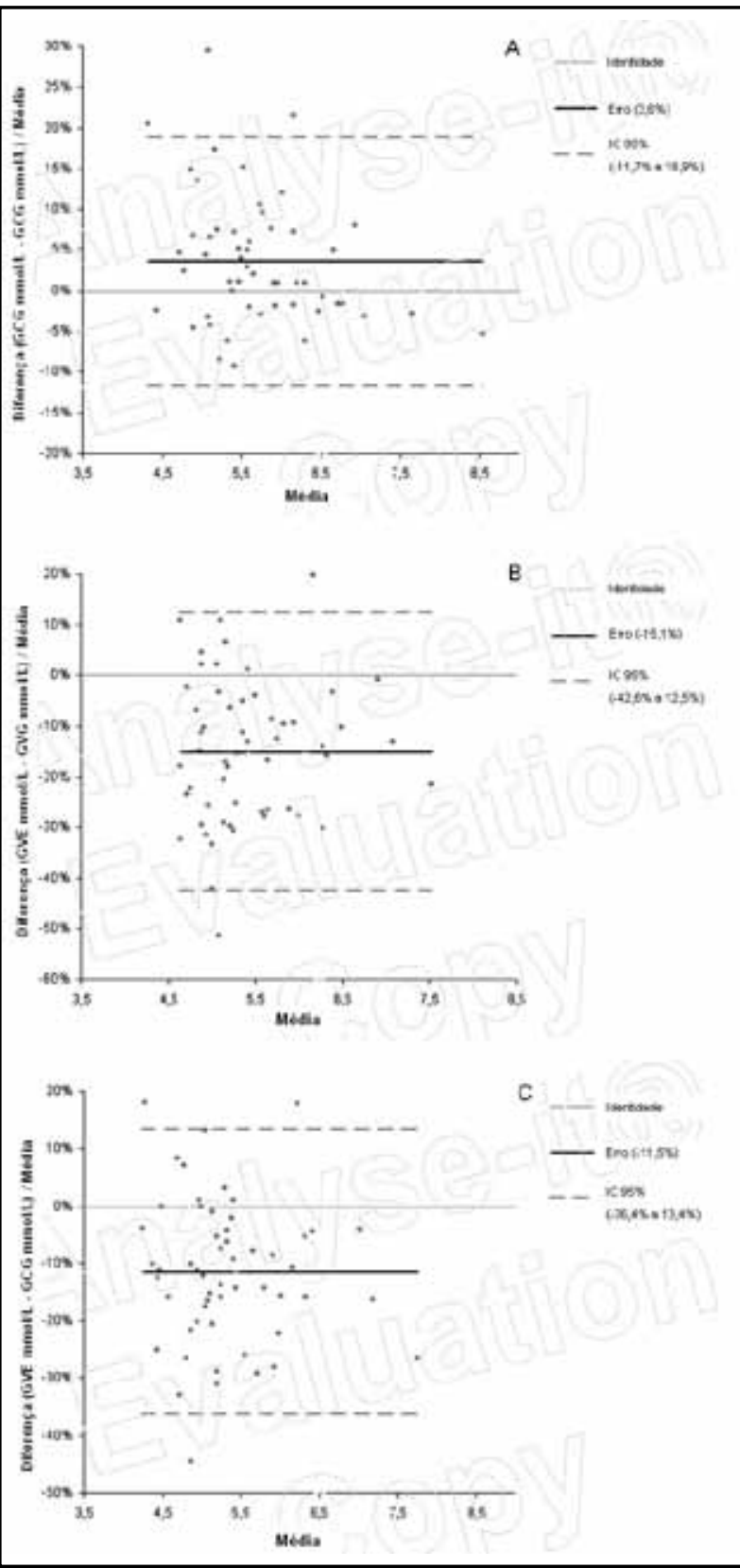

Figura 2 - A: Gráfico das diferenças de Bland-Altman entre os resultados da GCG e da GVG; B: entre a GVG e a GVE: C: entre a GCG e a GVE

GCG: glicemia capilar determinada pelo glicosímetro; GVG: glicemia venosa determinada pelo glicosímetro; GVE: glicemia venosa determinada em plasma pelo método enzimático.

cance dessas metas. O Diabetes Control and Complications Trial (DCCT) mostrou que o controle glicêmico estrito no DM1 reduz o desenvolvimento de retinopatia, nefropatia e, talvez, vasculopatia, comparativamente ao tratamento convencional. O United Kingdom Prospective Diabetes Study (UKPDS) demonstrou o mesmo em pacientes com DM2, enfatizando também a importância do tratamento da hipertensão e de outros fatores associados nesses $\operatorname{casos}^{(5)}$. Além disso, entre os fatores de risco para o desenvolvimento 


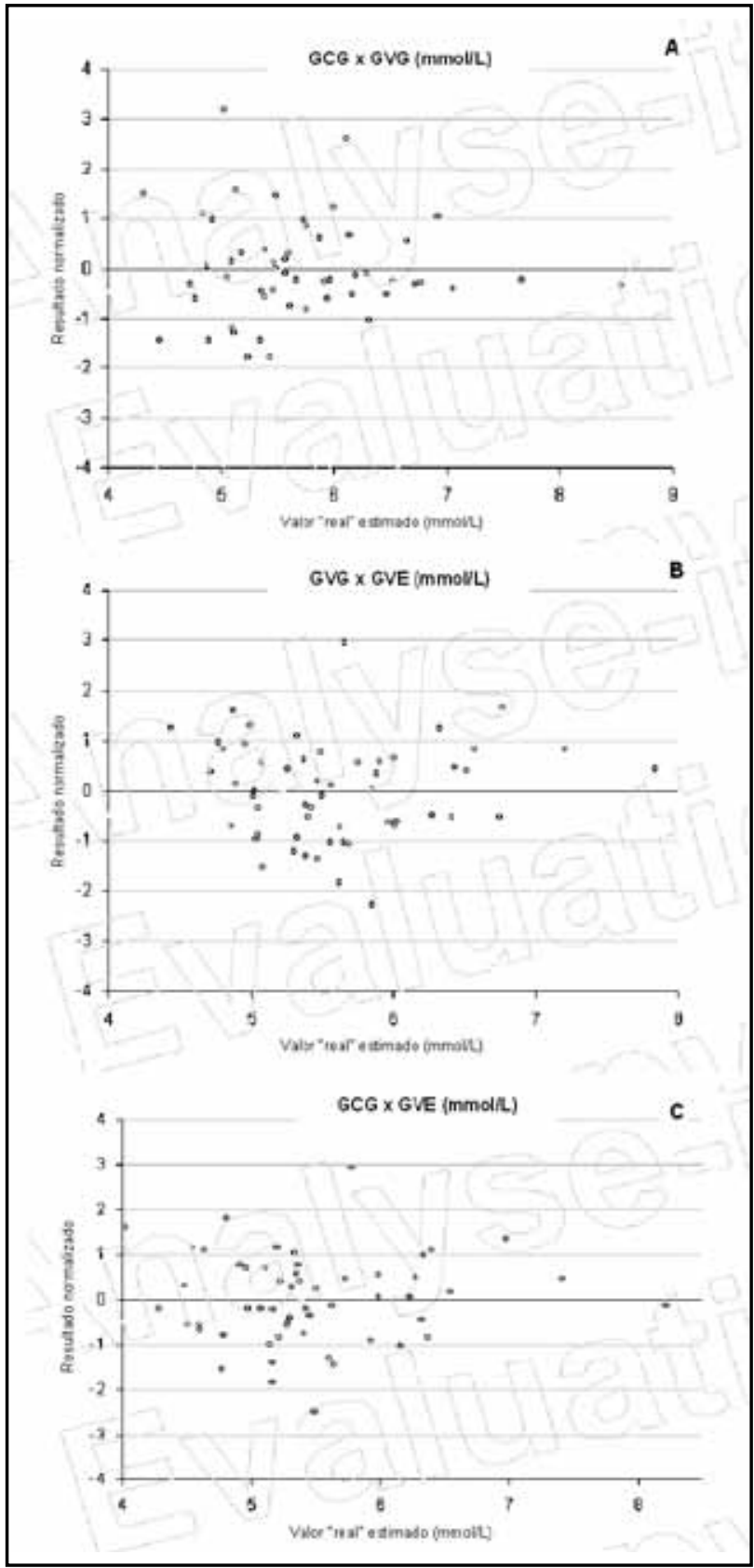

Figura 3 - A: Gráfico dos resultados normalizados derivados da regressão de Deming entre os valores da GCG e da GVG; B: entre a GVG e a GVE; C: entre a GCG e GVE, considerando apenas os pacientes com glicemia alterada

GCG: glicemia capilar determinada pelo glicosímetro; GVG: glicemia venosa determinada pelo glicosímetro; GVE: glicemia venosa determinada em plasma pelo método enzimático.

das doenças cardiovasculares (DCVs) encontra-se o DM, que apresenta alta taxa de morbimortalidade e perda na qualidade de vida. Constitui, ainda, uma das principais causas de insuficiência renal, amputação de membros inferiores e cegueira ${ }^{(14)}$.

Os avanços tecnológicos que permitiram o desenvolvimento de aparelhos para a avaliação remota da glicemia em sangue capilar, até pelo próprio paciente, têm repre- sentado um ganho significativo no controle da doença, especialmente quando fazem parte de um programa de saúde bem estruturado ${ }^{(13)}$.

Entretanto, além de os pacientes frequentemente questionarem as diferenças de resultados obtidos com os glicosímetros e os resultados da glicemia plasmática determinados pelos laboratórios, os estudos disponíveis apresentam dados bastante conflitantes. Por exemplo, alguns autores ${ }^{(3)}$ encontraram boa correlação entre a glicemia plasmática e a glicose capilar em dois tipos de glicosímetros, Optimum Xceed $(r=0,938)$ e One Touch Ultra $(r=0,911)$. Entretanto, de forma semelhante aos resultados encontrados em nosso estudo, a correlação foi pior para os pacientes normoglicêmicos. Em outro estudo, os resultados obtidos com Glucocard Memory 2 foram também comparáveis à glicemia plasmática ${ }^{(2)}$. Utilizando um equipamento de série diferente, mas da mesma marca do fabricante utilizada em nosso estudo, outros autores observaram ótima correlação entre a glicemia capilar obtida com o glicosímetro Accu-Check Compact e a glicemia plasmática determinada por método enzimático $(r=0,9819)^{(4)}$. Resultado semelhante foi encontrado com o mesmo equipamento por outro estudo no Brasil( ${ }^{(9)}$. Entretanto, como o primeiro grupo demonstrou, em pacientes graves o equipamento pode apresentar imprecisão significativa em relação à glicemia plasmática ${ }^{(4)}$. Já em outro estudo, avaliando o glicosímetro Medisense Precision Plus (Abbott), foi encontrada diferença significativa quanto à glicemia plasmática(1).

Utilizando-se exatamente o mesmo aparelho avaliado em nosso estudo (Accu-Check Advantage, Roche) em pacientes neonatos, observou-se que os resultados da glicemia capilar são superestimados em relação à glicemia

\begin{tabular}{|c|c|c|c|}
\hline \multirow[t]{2}{*}{ Tabela } & \multicolumn{3}{|c|}{$\begin{array}{l}\text { Resultados obtidos na determinação } \\
\text { da GCG, da GVG e da GVE }\end{array}$} \\
\hline & $\begin{array}{c}\text { GCG } \\
(\mathrm{mmol} / \mathrm{l})\end{array}$ & $\begin{array}{c}\text { GVG } \\
(\mathrm{mmol} / \mathrm{l})\end{array}$ & $\begin{array}{c}\text { GVE } \\
(\mathrm{mmol} / \mathrm{l})\end{array}$ \\
\hline $\begin{array}{l}\text { Faixa de } \\
\text { valores }\end{array}$ & $3,89-8,77$ & $4,38-8,33$ & $3,77-6,88$ \\
\hline Média & 5,64 & 5,83 & 5,02 \\
\hline Mediana & 5,49 & 5,72 & 4,94 \\
\hline $\begin{array}{l}\text { Desvio } \\
\text { padrão }\end{array}$ & 0,87 & 0,76 & 0,73 \\
\hline
\end{tabular}

GCG: glicemia capilar determinada pelo glicosímetro; GVG: glicemia venosa determinada pelo glicosímetro; GVE: glicemia venosa determinada em plasma pelo método enzimático. 
plasmática $^{(7)}$. Por outro lado, alguns autores não encontraram diferença significativa com esse mesmo glicosímetro em comparação com a glicemia plasmática, avaliando 20 amostras de pacientes submetidos a cirurgia cardíaca ${ }^{(6)}$.

Como se pode perceber, o fato de haver diferentes estudos com resultados conflitantes entre si não depende somente da marca ou série específica do glicosímetro, posto que uma mesma marca pode apresentar resultados inconsistentes em diferentes estudos. Normalmente, espera-se que os resultados da glicemia capilar sejam maiores que os da glicemia em plasma venoso ${ }^{(8)}$. Isso se deve não só ao tempo requerido para que alterações na glicose venosa alcancem os níveis do sangue capilar, mas também ao tipo de amostra utilizado. Os glicosímetros são calibrados para a utilização de sangue total. Assim, o sangue total venoso fornece resultados muito próximos aos do sangue total capilar, como observado em nossos resultados.

Entretanto, torna-se importante ressaltar que essas informações sobre a possível variabilidade de resultados de glicemia, quando se comparam os resultados da GCG com os da GVE fornecidos pelo laboratório, são frequentemente desconhecidas pela população e, muitas vezes, pelos profissionais da saúde responsáveis pelo atendimento aos pacientes diabéticos. É vital que todos os envolvidos nesse processo tenham informações corretas e consistentes para orientar os pacientes e que, de preferência, cada laboratório possa avaliar o desempenho dos glicosímetros utilizados como testes remotos em unidades de terapia intensiva (UTIs), enfermarias, emergências, e outros locais sob sua responsabilidade.

\section{Referências}

1. BOYD, R.; LEIGH, B.; STUART, P. Capillary versus venous bedside blood glucose estimations. Emerg Med J, v. 22, n. 3, p. 177-9, 2005.

2. CARRERA, T. et al. Evaluation of the Glucocard Memory 2 analyzer for measuring glucose concentration in capillary blood. Aten Primária, v. 26, n. 5, p. 327-30, 2000.

3. COYNE, S.; LACOUR, B.; HENNEQUIN-LE MEUR, C. Evaluation of Optium Xceed (Abbott) and One Touch Ultra (Lifescan) glucose meters. Ann Biol Clin, v. 66, n. 3, p. 249-54, 2008.

4. CRITCHELL, C. D. et al. Accuracy of bedside capillary blood glucose measurements in critically ill patients. Intensive Care Med, v. 33, n.12, p.2079-84, 2007

5. FUCHS, F. D; WANNMACHER, L.; FERREIRA, M. B. C. Farmacologia clínica: fundamentos da terapêutica racional. Rio de Janeiro: Guanabara Koogan, p. 1074, 2006.

6. KARON, B. S. et al. Accuracy of roche accu-chek inform whole blood capillary, arterial and venous glucose values in patients receiving intensive intravenous insulin therapy after cardiac surgery. Am J Clin Pathol, v. 127, n. 6, p. 919-26, 2007.

7. MCNAMARA, P. J.; SHARIEF, N. Comparison of EML 105 and advantage analysers measuring capillary versus venous whole blood glucose in neonates. Acta Paediatr, v. 90 , n. 9, p. 1033-41, 2001.
8. MELNIK, J.; POTTER, J. L. Variance in capillary and venous glucose levels during a glucose tolerance test. Am J Med Technol, v. 48, n. 6, p. 543-5, 1982.

9. MIRA, G. S.; CANDIDO, L. M.; YALE, J. F. Performance of glucometer used for self-monitoring blood glycaemia in type 1 diabetic patients. Arq Bras Endocrinol Metabol, v. 50, n. 3, p. 541-9, 2006

10. NCCLS, Method Comparison and Bias Estimation Using Patient Samples; Approved guideline second edition, NCCLS document EP09-A2, 2002.

11. PRADO, C. F.; RAMOS, J.; DO VALLE, R. Atualização terapêutica. São Paulo: Liv. Ed. Artes Médicas, p. $1820,2003$.

12. SACKS, D. B. et al. Guidelines and recommendations for laboratory analysis in the diagnosis and management of diabetes mellitus. Diabetes Care, v. 25, n. 4, p. 436-72, 2002.

13. SHEPHARD, M. D. et al. The impact of point of care testing on diabetes services along Victorias Mallee Track: results of a community-based diabetes risk assessment and management program. Rural Remote Health, v. 5, n. 3, p. 371, 2005.

14. Tavares, D. M. S. et al. Caracterização de idosos diabéticos atendidos na atenção secundária. Ciência e Saúde Coletiva, v. 12, n. 5, p. 1341-52, 2007. 